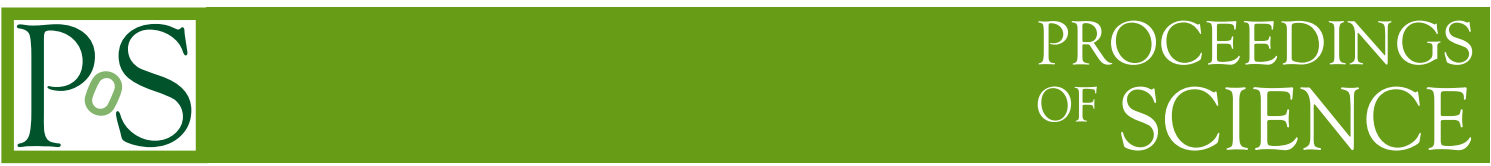

\title{
Exotic Higgs Searches at the LHC
}

\author{
Sara Diglio ${ }^{a}$ and Stefano Lacaprara ${ }^{b}$ \\ ${ }^{a}$ University of Melbourne, Melbourne, Australia \\ E-mail: Sara.Diglio@cern.ch \\ ${ }^{b}$ INFN sezione di Padova \\ E-mail: Stefano.Lacaprara@pd.infn.it
}

A review of the searches for exotic Higgs boson beyond standard model, from experiments at the LHC, is presented. The most recent results from ATLAS and CMS collaborations will be briefly summarized. These results are mostly based on specific models, including MSSM, nMSSM, SeeSaw and 2HDM. Two proposals for a more general interpretation of results, in term of generic 2HDM model and SM Higgs doublet plus an EW real singlet will be discussed.

LHCpp, VI Workshop Italiano sulla Fisica p-p a LHC

May 8-10 2013

Genova, Italy 


\section{Introduction}

The recent discovery of a new boson by ATLAS [1] and CMS [2] collaborations at the LHC is possibly the last missing stone of the Standard Model (SM) building. The most recent studies on its properties show that this boson is remarkably similar to the Higgs boson predicted by the SM $[3,4,5]$. However, the SM is well known to break at a larger scale and some major open points must be understood: the unification of couplings; the hierarchy problem; the dark matter issue; and the source of neutrino masses. In relation to these aspects, searches for new physics as an extended Higgs sector, with additional Higgs-like particles, are of great interest.

\section{Searches}

This report summarizes some of the searches performed at the Large Hadron Colliders (LHC) for an Higgs-like particle beyond the SM. Many analysis have been preformed by the ATLAS [6] and CMS [7] collaborations, considering a number of different scenarios.

A possible set of extensions of the SM, called Two Higgs Doublet Model [8] (2HDM), adds a second doublet in the Higgs sector, giving rise to a total of five physical states: three neutral $(\mathrm{h}, \mathrm{H}$ CP-even and A CP-odd) and two charged $\left(\mathrm{H}^{ \pm}\right)$. These models introduce two mixing angles $\alpha$ and $\beta$, which describe the mixing of CP-even and CP-odd states, respectively. Also, $\tan \beta$ is the ratio of the vacuum expectation values of the two doublets: $\tan \beta=v_{2} / v_{1}$. These general models can be further classified depending on the fermion coupling to the Higgs sector. In type I 2HDM models all quarks couple to just one of the Higgs doublets. These include the fermio-phobic limit scenario. If up-type quarks couple to one Higgs doublet and down-type quarks couple to the other, we have Type II models. A well known examples is the minimal supersymmetric standard model (MSSM). Other types (III and IV) consider different coupling for leptons and are not considered here.

\subsection{MSSM searches}

Many searches have considered the minimal supersymmetric standard model (MSSM). This particular declination of Type II $2 \mathrm{HDM}$ can be described by two parameters: $\tan \beta$ and $\mathrm{m}_{\mathrm{A}}$. For large values of $\tan \beta$ the coupling to b-quarks is enhanced with respect to that of the SM. The associate production $g g \rightarrow \mathrm{bb} \phi$ is favourite and the most important decay channels are $\phi \rightarrow \mathrm{bb} / \tau \tau / \mu \mu$ with BR 90/10/0.04\%, respectively. The most stringent limits obtained so far by both experiments are those using the $\tau$ final states. The CMS collaboration, using an integrated luminosity of $5 \mathrm{fb}^{-1}$ at $7 \mathrm{TeV}$ and $12 \mathrm{fb}^{-1}$ at $8 \mathrm{TeV}$, published a lower limit as low as $\tan \beta>5$ for $\mathrm{m}_{\mathrm{A}}<250 \mathrm{GeV}$ [9], and less stringent limit for $\mathrm{m}_{\mathrm{A}}$ up to $800 \mathrm{GeV}$. ATLAS has a similar analysis with less integrated luminosity ( 4.7 to $4.8 \mathrm{fb}^{-1}$ at $7 \mathrm{TeV}$ ) and, correspondingly, less stringent limits [10]. Both collaboration have also worked on the $\mu \mu$ final state, that, despite the small BR, has a very clean signature $[10,11]$. The limit reached is about $\tan \beta>10(20)$ for low $\mathrm{m}_{\mathrm{A}}$ for ATLAS with a total integrated luminosity of $\mathrm{L}=4.7-4.8 \mathrm{fb}^{-1}$ at $7 \mathrm{TeV}$ and $\mathrm{CMS}$ with $\mathrm{L}=5 \mathrm{fb}^{-1}$ at $7 \mathrm{TeV}$, respectively. CMS has also published an analysis using the $\phi \rightarrow$ bb decay, which suffers from the very large background from QCD multijets events, gluon splitting into heavy flavour jets, flavour excitation as well as from b-jet mis-identification. A limit on $\tan \beta>20$ for $\mathrm{m}_{\mathrm{A}} \sim 120 \mathrm{GeV}$ has been reached [12]. 
The charged Higgs $\mathrm{H}^{ \pm}$can be produced either from top quark decay $\left(\mathrm{t} \rightarrow \mathrm{bH}^{ \pm}\right)$if $\mathrm{m}_{\mathrm{h}^{ \pm}}<\mathrm{m}_{\text {top }}$ or via $\mathrm{gg} \rightarrow \mathrm{tbH}^{ \pm}$otherwise. In the first case, which has been investigated by ATLAS and CMS collaborations, the preferred decay channels are $\mathrm{H}^{ \pm} \rightarrow \tau^{ \pm} v$ and $\mathrm{H}^{ \pm} \rightarrow \mathrm{cs}$ which are dominant for large and small $\tan \beta$, respectively. Both semileptonic and hadronic decay of the associated top quark have been investigated in the $\mathrm{H}^{ \pm} \rightarrow \tau \nu$ channel. The analysis has been performed by ATLAS [13] and CMS [14] using only $7 \mathrm{TeV}$ data. A limit on the $\mathrm{BR}$ of $\mathrm{t} \rightarrow \mathrm{bH}^{ \pm}$of the order of few percent for $80<\mathrm{m}_{\mathrm{h}^{ \pm}}<160 \mathrm{GeV}$ have been reached. ATLAS has also considered the $\mathrm{H}^{ \pm} \rightarrow \mathrm{cs}$ channel, reaching a similar limit in BR [15].

\subsection{Next-to-MSSM searches}

The next-to MSSM (NMSSM) model [16] adds a further scalar singlet to the Higgs sector of the MSSM. It has some advantages over the MSSM, since it accommodates better for a lighter Higgs boson with a mass $m_{h}=125 \mathrm{GeV}$. Furthermore, it solves the problem of the $\mu$-term in the Lagrangian of the theory, since that term is dynamically produced by a vacuum expectation value of the singlet, avoiding the need of fine-tuning. The resulting Higgs sector in the NMSSM comprises three CP-even states $\mathrm{h}_{1,2,3}$, two CP-odd ones $\mathrm{a}_{1,2}$, and two charged $\mathrm{H}^{ \pm}$. The $\mathrm{a}_{1}$ can be very light $\mathrm{m}_{\mathrm{a}_{1}} \lesssim 2 \mathrm{~m}_{\mathrm{b}}$ and can have a significant branching ratio to photons, muons, taus, and bottom quarks for large $\tan \beta$, depending on the kinematically allowed decay channel for a given $a_{1}$ mass [17]. Some of these final states have been investigated at the LHC. Direct production of $\mathrm{a}_{1}$ and its decay into a pair of $\mu$ has been searched for with a dedicated exclusive trigger path, for pair of muons with invariant mass around the $\mathrm{Y}(\mathrm{nS})$ resonance $[18,19]$. Furthermore, ATLAS has considered the decay chain $\mathrm{h} \rightarrow \mathrm{aa} \rightarrow 4 \gamma$, where the resulting $\gamma$ pairs would be very collimated given the mass difference of $\mathrm{h}$ and a and the large boost of the latter boson [20]. For this final state, the limit reached is $\sigma(\mathrm{pp} \rightarrow \mathrm{h} \rightarrow$ aa $\rightarrow 4 \gamma)<0.1(0.2)$ pb for $\mathrm{m}_{\mathrm{A}}=100,200(400) \mathrm{MeV}$. CMS has instead studied a similar decay chain with $a \rightarrow \mu \mu$ decay [21]. A model independent limit on $\sigma \times B R \times$ Acceptance is set at $0.78 \pm 0.05 \mathrm{fb}$, corresponding to a limit on $\sigma(\mathrm{pp} \rightarrow \mathrm{h} \rightarrow \mathrm{aa} \rightarrow 4 \mu) \lesssim 1.5(3) \mathrm{pb}$ for $\mathrm{m}_{\mathrm{A}}=0.25(1$ or 3.55$) \mathrm{GeV}$ and $\mathrm{m}_{\mathrm{h}}=125 \mathrm{GeV}$.

\subsection{Other searches}

The ATLAS collaboration has analyzed the decay chain $\mathrm{H} \rightarrow \mathrm{WW} \rightarrow \mathrm{e} v \mu \nu$, within $2 \mathrm{HDM}$ scenarios, under the hypothesis that the state at $125 \mathrm{GeV}$ is the light Higgs (h) and that $\mathrm{A}$ and $\mathrm{H}^{ \pm}$ are heavy enough not to affect the cross section and branching ratio of $\mathrm{H}$ [22]. The null results have been interpreted in the $\mathrm{m}_{\mathrm{h}} \mathrm{vs} \cos \alpha$ plane for a set of values of $\tan \beta$.

Recent results from CMS on $\mathrm{h} \rightarrow \mathrm{WW} \rightarrow \ell v \mathrm{q} \overline{\mathrm{q}}$ [23] and on $\mathrm{h} \rightarrow \mathrm{ZZ} \rightarrow 2 \ell 2 v$ [24] have been interpreted in the context of heavier Higgs boson search, beyond the SM, using an alternative model, which add an electro-weak singlet to the SM Higgs sector. This model will be discussed later.

Other scenarios have been investigated at the LHC, including fermio-phobic (FP) Higgs and standard model with forth generation of fermions (SM4). The FP Higgs has been excluded up to $\mathrm{m}_{\mathrm{h}_{\mathrm{fp}}}<147 \mathrm{GeV}$ [25], while the SM4 Higgs is excluded in the range $100<\mathrm{m}_{\mathrm{h}_{\mathrm{SM} 4}}<600 \mathrm{GeV}[26$, 25].

A direct search of the invisible decay of Higgs by ATLAS, using associate production $\mathrm{Z} \rightarrow \mathrm{Zh}$, and setting an upper limit on $\mathrm{BR}(\mathrm{h} \rightarrow$ invisible $)<30 \%$ [27]. 
Finally, a direct search for a doubly charged Higgs-like state has been performed by both collaborations. The reference model is the minimal seesaw of type II [28] which includes in the SM Higgs sector an additional scalar field, that acts as a triplet under $\mathrm{SU}(2)_{\mathrm{L}}$. This triplet produces a set of Higgs boson-like particles: neutral, charged and doubly charged $\Phi^{0}, \Phi^{+}, \Phi^{++}$. The Yukawa coupling matrix elements of these scalar field are proportional to the light neutrino mass matrix. They decay into leptons, including flavour-violating decay. The $\Phi^{++}$decays into pair of samecharge leptons, for which the SM background is highly suppressed. Other models, such as Higgs triplets, little Higgs and Left-Right Symmetric models can be considered as well. The analysis of $7 \mathrm{TeV}$ data by ATLAS [29] and CMS [30] reached a limit in mass $\mathrm{m}_{\Phi^{++}}>200-450 \mathrm{GeV}$ depending on the final state considered.

\section{Future plans}

From the brief summary of the analysis published by the LHC collaborations, it is clear that many searches do not makes use of the $125 \mathrm{GeV}$ boson discovered in the context of SM Higgs searches. On the contrary, some analysis show an excess in correspondence of a mass $\mathrm{m}_{\mathrm{h}}=125 \mathrm{GeV}$. This is particularly evident in searches which are similar to that for the SM Higgs, or are a reinterpretation of that, e.g. fermio-phobic and SM4. The reason for this is of course that most of the analysis started well before the discovery of the $125 \mathrm{GeV}$ state. A more direct involvement of the $125 \mathrm{GeV}$ boson can extend the current searches for other Higgs states, by including the $\mathrm{h}_{125}$ in the decay chain considered. From the experimental point of view, having a resonance of known mass in the decay chain can certainly help the selection efficiency for a signal. For examples, the following new channels could be considered: $\mathrm{H} \rightarrow \mathrm{h}_{125} \mathrm{~h}_{125}, g g \rightarrow \mathrm{h}_{125} \rightarrow \mathrm{h}_{125} \mathrm{~h}_{2}, \mathrm{~A} \rightarrow \mathrm{h}_{125} \mathrm{Z}$, etc.

Also, the model considered does not explicitly have an Higgs state at $125 \mathrm{GeV}$, even though the MSSM inspired ones can easily accommodate it. The benchmark model widely used so far, the so called $\mathrm{m}_{\max }^{\mathrm{h}}$, was developed with the aim to allow the lightest Higgs to be as heavy as possible (hence the name), so it can have $\mathrm{m}_{\mathrm{h}}$ up to $\sim 135 \mathrm{GeV}$, thanks to radiative corrections. This is not needed anymore, so also a review of the benchmark model is advisable. A recent work has indeed introduced a modified version of the benchmark, called $\mathrm{m}_{\mathrm{mod}}^{\mathrm{h}}$, which takes into account the evidence of a boson at $125 \mathrm{GeV}$ [31]. With this revised benchmark model, the phase space compatible with the existence of a $125 \mathrm{GeV}$ state is increased with respect to the $\mathrm{m}_{\max }^{\mathrm{h}}$ one, where only a narrow band was allowed, as shown by the green regions figure 1 .

Furthermore, some recent studies indicates that, in the context of MSSM, the low $\tan \beta$ region is potentially of interest, in particular if the SUSY scale is not requested to be close to $1 \mathrm{TeV}$, as was the case for standard MSSM benchmark scenario considered so far [32]. Most of this region was excluded by LEP [33], and little extension to that limit has been provided by LHC: only the search $\mathrm{t} \rightarrow \mathrm{H}^{+} \rightarrow \mathrm{cs}$ has contributed [15] , while all other direct MSSM searches have sensitivity to high $\tan \beta$, the most sensitive being $\phi \rightarrow \tau \tau$. This low $\tan \beta$ region can be explored by heavier $H$ boson decaying to WW or ZZ, below the $\bar{t} \bar{t}$ decay threshold. Especially for $\mathrm{H} \rightarrow \mathrm{WW}$, which has a very poor resolution on the $\mathrm{H}$ boson mass if the leptonic decay of the $\mathrm{W}$ are used, the contribution of $\mathrm{h}_{125} \rightarrow \mathrm{WW}$ should be considered as background and taken into account, strange as it may seem. At higher masses, also the hadronic decay for at least one vector boson can be considered. It is important to note that the width of such an heavy higgs is narrow $\lesssim 3 \mathrm{GeV}$, since the coupling $g_{\mathrm{HVV}}$ 

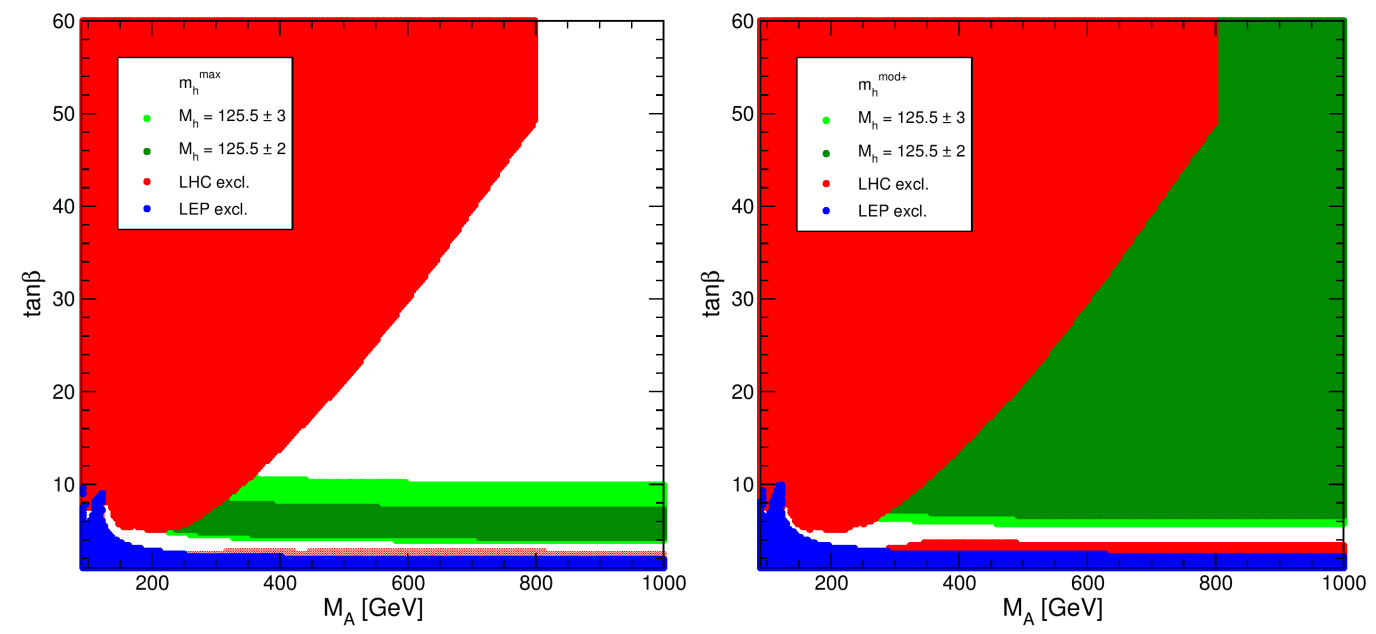

Figure 1: The $\tan \beta$ vs $\mathrm{m}_{\mathrm{A}}$ planes in the $\mathrm{m}_{\max }^{\mathrm{h}}$ (left) and $\mathrm{m}_{\text {mod }}^{\mathrm{h}}$ (right) scenario, with excluded regions from direct Higgs searches at LEP (blue), and the LHC (solid red); the dotted (lighter) red region is excluded by LHC searches for a SM-like Higgs boson. The two green shades correspond to the parameters for which $\mathrm{m}_{\mathrm{h}}=125 \pm 2(3) \mathrm{GeV}$. These figures are taken from [31]

is suppressed. At even higher mass, the $\mathrm{H} / \mathrm{A} \rightarrow \mathrm{t} \overline{\mathrm{t}}$ is relevant. Other channels which can explore the low $\tan \beta$ region are $\mathrm{A} \rightarrow \mathrm{Zh}_{125}$ and $\mathrm{H} \rightarrow \mathrm{h}_{125} \mathrm{~h}_{125}$. Figure 2, from [32], shows the expected sensitivity for these channels using a MSSM scenario where $\mathrm{m}_{\mathrm{h}}=125 \mathrm{GeV}$.

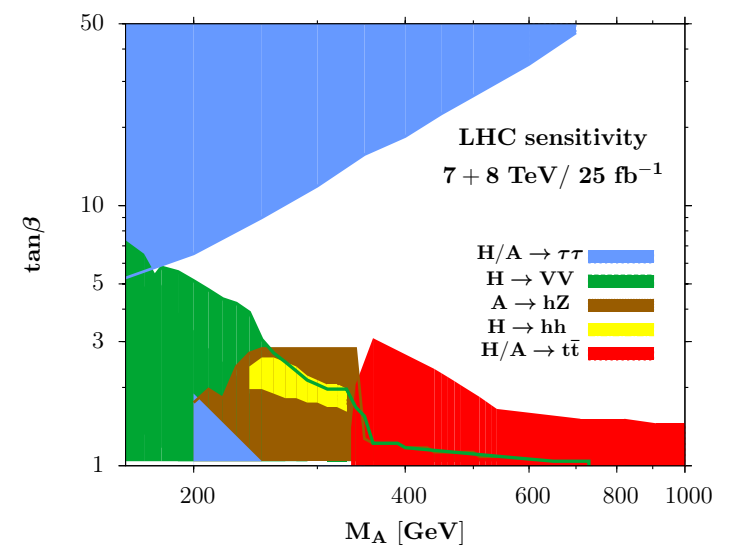

Figure 2: The estimated sensitivity for various channels for the heavier MSSM H boson in the $\tan \beta \mathrm{vs}_{\mathrm{A}}$ plane. This figure is taken from [32]

More generally, it would be interesting to try to develop some model-independent searches. This is quite difficult, since the phase space available in the Higgs sector, even with one Higgs found, is very large. However, a set of simple models, considered as effective theories, can be envisaged to provide benchmark point and a common interpretation of experimental results.

Any such model must be consistent with the observation of the boson at $125 \mathrm{GeV}$, contain an heavy Higgs-like state, and be compatible with the precision electroweak measurements available. Further assumption can be made, namely the spin and CP of the heavy state is $0^{+}$, the custodial symmetry between $\mathrm{W}$ and $\mathrm{Z}$ is not broken, and the new state does not introduce flavour changing 
neutral currents. Two methods have been proposed: one is a generic $2 \mathrm{HDM}$ model [8, 34], already used by ATLAS and discussed before, and one based on the addition of one electro-weak singlet to the SM Higgs sector [35, 36, 37], used recently by CMS to interpret the results of $\mathrm{H} \rightarrow \mathrm{WW} \rightarrow \ell v \mathrm{q} \overline{\mathrm{q}}$ and $\mathrm{H} \rightarrow \mathrm{ZZ} \rightarrow 2 \ell 2 v$ searches.

For the former model (2HDM), several choices of benchmark can be done, in addition to those presented by ATLAS in the $\mathrm{m}_{\mathrm{h}} \mathrm{vs} \cos \alpha$ for various values of $\tan \beta$. For example, a better choice would be $|\cos (\alpha-\beta)|$ (which correspond to the HWW coupling) vs $\tan \beta$, for various choices of $\lambda, \lambda_{A}$ and $\lambda_{F}$ (linear combination of the Higgs self-coupling), limiting $|\cos (\alpha-\beta)|$ between 0 and 0.5 , corresponding to a very rough SM-like $\mathrm{h}_{125}$. An alternative would be a three parameters scan over $|\cos (\alpha-\beta)|, \tan \beta$ and $\mathrm{m}_{\mathrm{h}}$ and choose benchmark values for $\lambda_{A}$ and $\lambda_{F}$.

In the latter model, SM+EW-singlet, the EW singlet introduces a second, heavier Higgs state (H). The coupling strength of the $\mathrm{h}$ and $\mathrm{H}$ to fermions and vectors are those predicted by the SM but they are rescaled by a factor $C$ and $C^{\prime}$, for $\mathrm{h}$ and $\mathrm{H}$, respectively. Under these assumptions, the unitarity condition requires that $C^{2}+C^{2}=1$. By considering the simplest case in which a possible $H \rightarrow h h$ decay is ignored, two constants can be rewritten as $C=\cos \theta$ and $C^{\prime}=\sin \theta$, where $\theta$ is, together with $\mathrm{m}_{\mathrm{h}}$, one of the two parameters of the theory. A further extension is to introduce a new branching ratio for the decays of the heaviest Higgs into unknown final states, including $\mathrm{H} \rightarrow \mathrm{hh}$. This introduces a third free parameter $B R_{\text {new }}$ and extend the generality of the model. In particular, the width of the heavy Higgs state becomes $\Gamma^{\prime}=\Gamma_{S M} \frac{C^{2}}{1-B R_{\text {new }}}$, so the state can be either very narrow or very wide. The signal strength instead scales by a factor $\mu^{\prime}=C^{\prime 2}\left(1-B R_{\text {new }}\right)$. The SM benchmark correspond to the limit $C^{\prime} \rightarrow 0$. The model can be explored in the plane $B R_{\text {new }}$ vs $C^{2}$, as shown in Fig. 3.

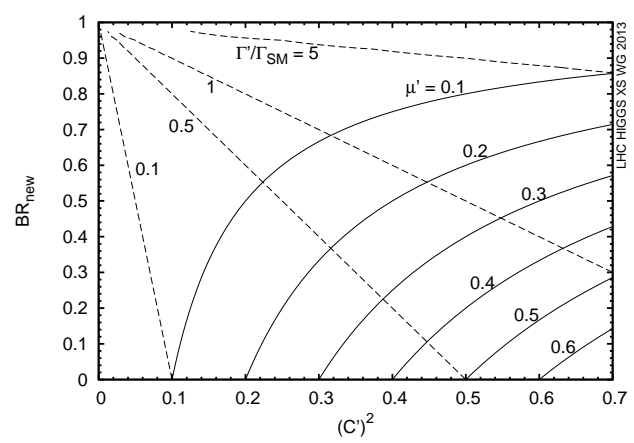

Figure 3: The parameters plane $B R_{\text {new }}$ vs $C^{\prime 2}$ of the SM plus EW-singlet model, with the isocurves for equal signal strength $\mu^{\prime}$ and width $\Gamma^{\prime} / \Gamma_{S M}$ (relative to SM one) of the heavier Higgs state.

The direct measurements of the signal strength of $h_{125}$ can be interpreted as a constraint on that of the $\mathrm{H}$ [37]. The current measurements on SM signal strength $\mu\left(\mu_{h}^{A T L A S}=1.3 \pm 0.2\right.$ [4]; $\mu_{h}^{C M S}=0.8 \pm 0.14$ [3]) can be interpreted to out lower bound on $\mu$ itself, and hence an upper bound on $\mu^{\prime}$. Taking the uncertainty to be Gaussian distributed, these correspond to a $2 \sigma$ lower limit of $\mu^{\prime 2}<0.1(0.46)$ for ATLAS(CMS), respectively. If $B R_{\text {new }}$ is assumed to be negligible, the upper bound is transferred directly on the heavy Higgs coupling: $C^{\prime 2}<0.1(0.46)$ for ATLAS(CMS). 


\section{Conclusion}

We briefly described the search for Higgs bosons beyond the standard model performed at the LHC by ATLAS and CMS collaborations and discussed some of the future plans as well as two possible scenarios for interpretation of current and new results with more generic benchmark models.

\section{References}

[1] ATLAS Collaboration, Observation of a new particle in the search for the Standard Model Higgs boson with the ATLAS detector at the LHC, Phys. Lett. B 716 (2012) 1 [1207. 7214].

[2] CMS Collaboration, Observation of a new boson at a mass of $125 \mathrm{GeV}$ with the CMS experiment at the LHC, Phys. Lett. B 716 (2012) 30 [1207 . 7235].

[3] CMS Collaboration, Combination of standard model Higgs boson searches and measurements of the properties of the new boson with a mass near 125 GeV, CMS-PAS-HIG-13-005, 2013.

[4] ATLAS Collaboration, Combined coupling measurements of the Higgs-like boson with the ATLAS detector using up to $25 \mathrm{fb}^{-1}$ of proton-proton collision data, ATLAS-CONF-2013-034, Mar, 2013.

[5] ATLAS Collaboration, Study of the spin of the new boson with up to $25 \mathrm{fb}^{-1}$ of ATLAS data, ATLAS-CONF-2013-040, Apr, 2013.

[6] ATLAS Collaboration, The ATLAS Experiment at the CERN Large Hadron Collider, JINST 3 (2008), no. $08 \mathrm{~S} 08003$.

[7] CMS Collaboration, The CMS experiment at the CERN LHC, JINST 3 (2008) S08004.

[8] G. Branco, P. Ferreira, L. Lavoura, M. Rebelo, M. Sher et. al., Theory and phenomenology of two-Higgs-doublet models, Phys.Rept. 516 (2012) 1-102 [1106.0034].

[9] CMS Collaboration, Higgs to tau tau (MSSM) (HCP), CMS-PAS-HIG-12-050, 2012.

[10] ATLAS Collaboration, Search for the neutral higgs bosons of the minimal supersymmetric standard model in pp collisions at $\sqrt{s}=7$ tev with the atlas detector, Journal of High Energy Physics 2013 (2013), no. 2.

[11] CMS Collaboration, Search for Neutral MSSM Higgs Bosons in the $\mu^{+} \mu^{-}$final state with the CMS experiment in pp Collisions at $\sqrt{s}=7 \mathrm{TeV}$, CMS-PAS-HIG-12-011, 2012.

[12] CMS Collaboration, Search for a Higgs boson decaying into a b-quark pair and produced in association with b quarks in proton-proton collisions at 7 TeV, CMS-HIG-12-033, Feb, 2013.

[13] ATLAS Collaboration, Search for charged higgs bosons decaying via $h^{ \pm} \rightarrow \tau v$ in $t \bar{t}$ events using $p p$ collision data at $\sqrt{s}=7$ tev with the atlas detector, Journal of High Energy Physics 2012 (2012), no. 6.

[14] CMS Collaboration, Updated search for a light charged Higgs boson in top quark decays in pp collisions at $\sqrt{s}=7 \mathrm{TeV}$, CMS-PAS-HIG-12-052, 2012.

[15] ATLAS Collaboration, Search for a light charged Higgs boson in the decay channel $H^{+} \rightarrow c \bar{s}$ in ttbar events using pp collisions at $\sqrt{s}=7 \mathrm{TeV}$ with the ATLAS detector, 1302.3694 .

[16] M. Maniatis, The Next-to-Minimal Supersymmetric Extension of the Standard Model reviewed, Internat. J. Modern Phys. A25 (2010), no. 18n19 3505-3602. 
[17] B. A. Dobrescu and K. T. Matchev, Light axion within the next-to-minimal supersymmetric standard model, JHEP 2000 (2000), no. 931.

[18] CMS Collaboration, Search for a light pseudoscalar boson in the dimuon channel, CMS-PAS-HIG-12-004, 2012.

[19] ATLAS Collaboration, A Search for a Light CP-Odd Higgs Boson Decaying to $\mu^{+} \mu^{-}$in ATLAS, ATLAS-CONF-2011-020, 2011.

[20] ATLAS Collaboration, Search for a Higgs boson decaying to four photons through light CP-odd scalar coupling using $4.9 \mathrm{fb}^{-1}$ of $7 \mathrm{TeV}$ pp collision data taken with ATLAS detector at the LHC, ATLAS-CONF-2012-079, Jul, 2012.

[21] CMS Collaboration, Search for a non-standard-model Higgs boson decaying to a pair of new light bosons in four-muon final states. Search for new light bosons in decays of a non-standard-model Higgs boson to two pairs of muons in proton-proton collisions at $\sqrt{s}=7 \mathrm{TeV}$, CMS-EXO-12-012, Oct, 2012.

[22] ATLAS Collaboration, Search for Higgs bosons in Two-Higgs-Doublet models in the $H \rightarrow W W \rightarrow e v \mu v$ channel with the ATLAS detector, ATLAS-CONF-2013-027, Mar, 2013.

[23] CMS Collaboration, Search for a Standard Model-like Higgs boson decaying into WW $\rightarrow \ell v q \bar{q}$ in pp collisions at $\sqrt{s}=8 \mathrm{TeV}$, CMS-PAS-HIG-13-008, 2013.

[24] CMS Collaboration, Search for a heavy Higgs boson in the H to ZZ to 2l2nu channel in pp collisions at $\sqrt{s}=7$ and $8 T e V$, Tech. Rep. CMS-PAS-HIG-13-014, CERN, Geneva, 2013.

[25] CMS Collaboration, Searches for Higgs bosons in pp collisions at $\sqrt{s}=7$ and 8 TeV in the context of four-generation and fermiophobic models, CMS-HIG-12-013, Feb, 2013.

[26] ATLAS Collaboration, Update of the Combination of Higgs Boson Searches in pp Collisions at $\sqrt{s}=7 T e V$ with the ATLAS Experiment at the LHC, ATLAS-CONF-2011-135, Sep, 2011.

[27] ATLAS Collaboration, Search for invisible decays of a Higgs boson produced in association with a $Z$ boson in ATLAS, ATLAS-CONF-2013-011, Mar, 2013.

[28] M. Magg and C. Wetterich, Neutrino mass problem and gauge hierarchy, Phys. Lett. B 94 (1980), no. $161-64$.

[29] ATLAS Collaboration, Search for doubly-charged Higgs bosons in like-sign dilepton final states at $\sqrt{s}=7$ TeV with the ATLAS detector, Eur.Phys.J. C72 (2012) 2244 [1210 . 5070 ].

[30] CMS Collaboration, Inclusive search for doubly charged Higgs in leptonic final states with the 2011 data at 7TeV, CMS-PAS-HIG-12-005, 2012.

[31] M. Carena, S. Heinemeyer, O. Stål, C. Wagner and G. Weiglein, MSSM Higgs Boson Searches at the LHC: Benchmark Scenarios after the Discovery of a Higgs-like Particle, 1302 . 7033.

[32] A. Djouadi and J. Quevillon, The MSSM Higgs sector at a high $M_{S U S Y}$ : reopening the low tan $\beta$ regime and the search for heavy Higgsses, 1304.1787.

[33] LEP Collaborations, Search for neutral mssm higgs bosons at lep, The European Physical Journal C Particles and Fields 47 (2006), no. 3 547-587.

[34] A. Djouadi, The Anatomy of electro-weak symmetry breaking. II. The Higgs bosons in the minimal supersymmetric model, Phys.Rept. 459 (2008) 1-241 [hep-ph/ 0503173 ]. 
[35] V. Barger, P. Langacker, M. McCaskey, M. J. Ramsey-Musolf and G. Shaughnessy, CERN LHC phenomenology of an extended standard model with a real scalar singlet, Phys. Rev. D 77 (Feb, 2008) 035005.

[36] M. T. Bowen, Y. Cui and J. D. Wells, Narrow trans-TeV Higgs bosons and $H \rightarrow$ hh decays: two LHC search paths for a hidden sector Higgs boson, Journal of High Energy Physics 2007 (2007), no. 03 036.

[37] LHC Higgs Cross Section Working Group, Handbook of LHC Higgs Cross Sections: 3. Higgs properties, to be published as CERN Yellow Report, 2013. 